\title{
Security Enhanced Dynamic ID based Remote User Authentication Scheme for Multi-Server Environments
}

\author{
Jun-Sub Kim ${ }^{1}$ and Jin $\mathrm{Kwak}^{2 *}$ \\ ${ }^{1}$ IT Convergence Research Institute, Sungkyunkwan University, Korea \\ ${ }^{2}$ Department of Information and Compute Engineering, Ajou University, Korea \\ ${ }^{1}$ jskim.isaa@gmail.com, ${ }^{2}$ security@ajou.ac.kr
}

\begin{abstract}
Multi-server environments are that the user registers the single registration server and since the user uses the service to authenticate on multi-server. For this, many user authentication schemes have been proposed for multi-server environments. In 2013, Li, et al., proposed dynamic ID based remote user authentication scheme for multi-server environments. Unfortunately, their scheme is vulnerable to forgery attacks and replay attacks. In this paper, we analyze the security vulnerabilities of Li et al.'s scheme, and propose dynamic ID based remote user authentication scheme for multi-server environments. The proposed scheme ensures the safety to various attacks such as forgery attacks and replay attacks. Just like the existing schemes, our scheme is efficient at using the hash function and exclusive-OR operation.
\end{abstract}

Keywords: Dynamic ID, Mutual authentication, Password, Multi-Server Environment

\section{Introduction}

User authentication is used to authenticate a legitimate user through an insecure channel. According to the use environment, user authentication can be divided into user authentication in single-server environments and user authentication in multi-server environments. For user authentication in multi-server environments, users can register at a registration center to access a server that is associated with the registration center. Many user authentication schemes have been proposed for multi-server environments [1-12].

In 2009, Hsiang and Shih proposed a secure dynamic-ID-based remote user authentication scheme for multi-server environments [7]. They claimed that their scheme is secure and more efficient than Liao and Wang's scheme. Unfortunately, Lee, et al., pointed out that Hsiang and Shih's scheme is vulnerable to masquerade attacks and server spoofing attacks, is not easily reparable, and cannot provide mutual authentication [8]. To solve these problems, Lee, et al., proposed an improvement to Hsiang and Shih's scheme. Recently, Li, et al., showed that Lee, et al.'s scheme does not provide authentication and is vulnerable to forgery attacks and server spoofing attacks [9]. In addition, Li et al., proposed a new scheme based on Lee, et al.'s scheme. However, their scheme is vulnerable to forgery attacks and replay attacks. In this paper, we analyze the security vulnerabilities of $\mathrm{Li}$, et al.'s dynamic-ID-based remote user authentication scheme and then we propose a security enhanced dynamic ID based remote user authentication scheme for multi-server environments.

This study is organized as follows: Section 2 describes a brief review of Li, et al.'s scheme, and Section 3 analyze the security vulnerabilities of Li et al.'s scheme. In Section 4 , we propose a security enhanced dynamic ID based remote user authentication scheme for multi-server environments. In Section 5, we analyze our proposed scheme of the security requirements and performance. Finally, Section 6 presents our conclusion. 


\section{Review of Li, et al.'s Scheme}

\section{Registration Phase}

Step R1. $U_{i} \rightarrow R C:\left\{I D_{i}, A_{i}\right\}$

The user $U_{i}$ selects identity $I D_{i}$ and password $P W_{i}$ and computes $A_{i}=h\left(b \oplus P W_{i}\right)$, where $b$ is a random number generated by $U_{i} \cdot U_{i}$ sends $I D_{i}$ and $A_{i}$ to $R C$ through a secure channel.

Step R2. After receiving a registration message from $U_{i}, R C$ computes $h(x \| y)$ and $h\left(S I D_{j} \| h(y)\right)$ using $x, y$, and $S I D_{j}$, where $x$ and $y$ are the master secret key and a secret number selected by $R C$, and $S I D{ }_{j}$ is the identity of the server.

Step R3. $R C \rightarrow U_{i}:\left\{\right.$ smart $\left.\operatorname{card}\left[C_{i}, D_{i}, E_{i}, h(\cdot), h(y)\right]\right\}$

$R C$ sends $h(x \| y)$ and $h\left(S I D_{j} \| h(y)\right)$ to server $S_{j}$ through a secure channel and computes $\quad B_{i}=h\left(I D_{i} \| x\right), \quad C_{i}=h\left(I D_{i}\|h(y)\| A_{i}\right), \quad D_{i}=h\left(B_{i} \| h(x \| y)\right), \quad$ and $\quad E_{i}=$ $B_{i} \oplus h(x \| y)$. Then $R C$ issues the smart card containing $\left[C_{i}, D_{i}, E_{i}, h(\cdot), h(y)\right]$ and delivers it to $U_{i}$ through a secure channel.

Step R4. $U_{i}$ enters $b$ into his/her smart card, and the smart card contains $\left[C_{i}, D_{i}\right.$, $\left.E_{i}, b, h(\cdot), h(y)\right]$.

\section{Login phase}

Step L1. The user $U_{i}$ inserts his/her smart card into the device and inputs $I D_{i}$ and $P W_{i}$. Then the smart card computes $A_{i}=h\left(b \oplus P W_{i}\right)$ and $C_{i}^{*}=h\left(I D_{i}\|h(y)\| A_{i}\right)$ and checks whether $C_{i}^{*}$ is equal to $C_{i}$. If they are not equal, the procedure is terminated.

Step L2. Otherwise, the smart card generates a random nonce $N_{i}$ and computes $P_{i j}=E_{i} \oplus h\left(h\left(S I D_{j} \| h(y)\right) \| N_{i}\right) \quad, \quad C I D_{i}=A_{i} \oplus h\left(D_{i}\left\|S I D_{j}\right\| N_{i}\right)$ $M_{1}=h\left(P_{i j}\left\|C I D_{i}\right\| D_{i} \| N_{i}\right)$, and $M_{2}=h\left(S I D_{j} \| h(y)\right) \oplus N_{i}$.

Step L3. $U_{i} \rightarrow S_{j}:\left\{P_{i j}, C I D_{i}, M_{1}, M_{2}\right\}$ $U_{i}$ sends $P_{i j}, C I D_{i}, M_{1}$, and $M_{2}$ to $S_{j}$.

\section{Verification Phase}

Step V1. After receiving the login request message from $U_{i}, S_{j}$ computes $N_{i}=$ $h\left(S_{j}\left\|_{j}\right\| h(y)\right) \oplus M_{2} \quad, \quad E_{i}=P_{i j} \oplus h\left(h\left(S_{j} D_{j} \| h(y)\right) \| N_{i}\right) \quad, \quad B_{i}=E_{i} \oplus h(x \| y) \quad, \quad D_{i}=$ $h\left(B_{i} \| h(x \| y)\right), \quad A_{i}=C I D_{i} \oplus h\left(D_{i}\left\|S I D_{j}\right\| N_{i}\right)$, and $M_{1}^{*}=h\left(P_{i j}\left\|C I D_{i}\right\| D_{i} \| N_{i}\right)$. Then, $S_{j}$ checks whether $M_{1}^{*}$ is equal to $M_{1}$. If they are equal, $S_{j}$ accepts the login request to $U_{i}$.

Step V2. Then, $S_{j}$ generates a random nonce $N_{j}$ and computes $M_{3}=h\left(D_{i}\left\|A_{i}\right\|\right.$ $\left.N_{j} \| S I D_{j}\right)$ and $M_{4}=A_{i} \oplus N_{i} \oplus N_{j}$, and sends $M_{3}$ and $M_{4}$ to $U_{i}$. 
Step V3. After receiving the authentication message from $S_{j}, U_{i}$ computes $N_{j}=A_{i}$ $\oplus N_{i} \oplus M_{4}$ and $M_{3}^{*}=h\left(D_{i}\left\|A_{i}\right\| N_{j} \| S I D_{j}\right)$. Then, $U_{i}$ checks whether $M_{3}^{*}$ is equal to $M_{3}$. If they are equal, $U_{i}$ authenticates $S_{j}$. Next, $U_{i}$ computes $M_{5}=h\left(D_{i}\left\|A_{i}\right\|\right.$ $N_{i} \| S I D_{j}$ ) and sends $M_{5}$ to $S_{j}$.

Step V4. After receiving the mutual authentication message from $U_{i}, S_{j}$ computes $M_{5}^{*}=h\left(D_{i}\left\|A_{i}\right\| N_{i} \| S I D_{j}\right)$ and checks whether $M_{5}^{*}$ is equal to $M_{5}$. If they are equal, the procedure is terminated. Otherwise, $S_{j}$ authenticates $U_{i}$, and the mutual authentication is completed.

After the mutual authentication, $U_{i}$ and $S_{j}$ computes $S K=h\left(D_{i}\left\|A_{i}\right\| N_{i}\left\|N_{j}\right\| S I D_{j}\right)$ for future secure communication.

\section{Vulnerability of Li, et al.'s Scheme}

\section{Forgery Attack}

Assume that an attacker $Z$ is a legal user of the system, stolen $U_{i}$ 's smart card, and eavesdrops the communication between $U_{i}$ and $S_{j}$. Then, $Z$ extracts the parameters $\left[C_{i}, D_{i}, E_{i}, b_{i}, h(\cdot), h(y)\right] \quad$ stored in $U_{i}$ 's smart card and computes $N_{i}=$ $h\left(S I D_{j} \mid h(y)\right) \oplus M_{2}$ and $A_{i}=C I D_{i} \oplus h\left(D_{i}\left\|S I D_{j}\right\| N_{i}\right)$.

Step 1. $Z$ inserts his/her smart card into the device and inputs $I D_{z}$ and $P W_{z}$. The smart card computes $A_{z}=h\left(b_{z} \oplus P W_{z}\right)$ and $C_{z}^{*}=h\left(I D_{z}\|h(y)\| A_{z}\right)$ and checks whether $C_{z}^{*}$ is equal to $C_{z} \cdot Z$ generates a random number $N_{z}$ and computes $P_{i k}=E_{i}$ $\oplus h\left(h\left(S I D_{k} \| h(y)\right) \| N_{z}\right), \quad C I D_{i}=A_{i} \oplus h\left(D_{i}\left\|S I D_{k}\right\| N_{z}\right), \quad M_{1}=h\left(P_{i k}\left\|C I D_{i}\right\| D_{i} \| N_{z}\right)$, and $M_{2}=h\left(S I D_{k} \| h(y)\right) \oplus N_{z}$.

Step 2. Then, $Z$ sends the forged login request message $\left\{P_{i k}, C I D_{i}, M_{1}, M_{2}\right\}$ to server $S_{k}$.

Step 3. After receiving the login request message $\left\{P_{i k}, C I D_{i}, M_{1}, M_{2}\right\}, S_{k}$ computes $N_{z}=h\left(S I D_{k} \| h(y)\right) \oplus M_{2}, \quad E_{i}=P_{i k} \oplus h\left(h\left(S I D_{k} \| h(y)\right) \| N_{z}\right), \quad B_{i}=E_{i} \oplus h(x \| y), \quad D_{i}=$ $h\left(B_{i} \| h(x \| y)\right), \quad A_{i}=C I D_{i} \oplus h\left(D_{i}\left\|S I D_{k}\right\| N_{z}\right), \quad$ and $\quad M_{1}^{*}=h\left(P_{i k}\left\|C I D_{i}\right\| D_{i} \| N_{z}\right)$, and checks whether $M_{1}^{*}$ is equal to $M_{1}$. If they are equal, $S_{k}$ accepts the login request to $Z$.

Step 4. $S_{k}$ generates a random nonce $N_{k}$, computes $M_{3}=h\left(D_{i}\left\|A_{i}\right\| N_{k} \| S I D_{k}\right)$ and $M_{4}=A_{i} \oplus N_{z} \oplus N_{k}$, and sends $M_{3}$ and $M_{4}$ to $Z$.

Step 5. After receiving the authentication message $\left\{M_{3}, M_{4}\right\}, Z$ computes $N_{k}=M_{4}$ $\oplus A_{i} \oplus N_{z} \quad$ and $\quad M_{3}^{*}=h\left(D_{i}\left\|A_{i}\right\| N_{k} \| S I D_{k}\right)$ and authenticates $S_{k}$ because $M_{3}^{*}$ is equal to $M_{3}$. Then, $Z$ computes $M_{5}=h\left(D_{i}\left\|A_{i}\right\| N_{z} \| S I D_{k}\right)$ and sends $M_{5}$ to $S_{k}$.

Step 6. After receiving the mutual authentication message $\left\{M_{5}\right\}, S_{k}$ computes $M_{5}^{*}=h\left(D_{i}\left\|A_{i}\right\| N_{z} \| S I D_{k}\right)$ and checks whether $M_{5}^{*}$ is equal to $M_{5}$. If they are equal, 
$S_{k}$ authenticates $Z$.

Therefore, Li, et al. 's scheme is vulnerable to forgery attack.

\section{Replay Attack}

Assume that an attacker $Z$ eavesdrops the communication message $\left\{P_{i j}, C I D_{i}, M_{1}\right.$, $\left.M_{2}, M_{3}, M_{4}, M_{5}\right\}$ between $U_{i}$ and $S_{j}$. Then, $Z$ sends the previous login request message $\left\{P_{i j}, C I D_{i}, M_{1}, M_{2}\right\}$ to $S_{j}$.

Step 1. After receiving the login request message $\left\{P_{i j}, C I D_{i}, M_{1}, M_{2},\right\}, S_{j}$ computes $N_{i}=h\left(S_{j} \| h(y)\right) \oplus M_{2} \quad, \quad E_{i}=P_{i j} \oplus h\left(h\left(S I D_{j} \| h(y)\right) \| N_{i}\right) \quad, \quad B_{i}=E_{i} \oplus h(x \| y) \quad$, $D_{i}=h\left(B_{i} \| h(x \| y)\right), \quad A_{i}=C I D_{i} \oplus h\left(D_{i}\left\|S I D_{j}\right\| N_{i}\right)$, and $M_{1}^{*}=h\left(P_{i j}\left\|C I D_{i}\right\| D_{i} \| N_{i}\right)$ and checks whether $M_{1}^{*}$ is equal to $M_{1}$. If they are equal, $S_{j}$ accepts the login request to $Z$.

Step 2. $S_{j}$ generates a random nonce $N_{j}^{*}$ and computes $M_{3}=h\left(D_{i}\left\|A_{i}\right\| N_{j}^{*} \| S I D_{j}\right)$ and $M_{4}=A_{i} \oplus N_{z} \oplus N_{j}^{*}$.

Step 3. Then, $S_{j}$ sends $M_{3}$ and $M_{4}$ to $Z$.

Step 4. After receiving the authentication message $\left\{M_{3}, M_{4}\right\}, Z$ checks whether $M_{3}^{*}$ is equal to $M_{3}$, where $M_{3}^{*}$ is the same value with received $M_{3}$ from $S_{j}$. If they are equal, $Z$ authenticates $S_{j}$ and sends $M_{5}$ to $S_{j}$.

Step 5. After previously receiving the mutual authentication message $\left\{M_{5}\right\}, S_{j}$ computes $M_{5}^{*}=h\left(D_{i}\left\|A_{i}\right\| N_{i} \| S I D_{j}\right)$ and checks whether $M_{5}^{*}$ is equal to $M_{5}$. If they are equal, $S_{j}$ authenticates $Z$.

Therefore, $\mathrm{Li}$, et al.'s scheme is vulnerable to replay attacks.

\section{The Proposed Scheme}

In this section, we propose security enhanced dynamic ID based remote user authentication scheme for multi-server environments. Registration center $R C$ selects the master secret key $x$ and a secret number $y$, computes $h(x \| y)$ and $h\left(S_{S}{ }_{j} \| h(y)\right)$, and then shares them with server $s_{j}$ though a secure channel. This scheme consists of four phases: registration phase, login phase, verification phase, and password change phase.

\section{Notation}

Table 1 shows the notation used describe our proposed scheme. 
Table 1. Notation of or Scheme

\begin{tabular}{|l|l|}
\hline Notation & Description \\
\hline$U_{i}$ & The ith user \\
\hline$S_{j}$ & The jth server \\
\hline$R C$ & Registration center \\
\hline$I D_{i}$ & Identity of $U_{i}$ \\
\hline$P W_{i}$ & Password of $U_{i}$ \\
\hline$S I D_{j}$ & Identity of $S_{j}$ \\
\hline$C I D_{i}$ & Dynamic ID of $U_{i}$ \\
\hline$x$ & Master secret key of registration center \\
\hline$y$ & Secret number of registration center \\
\hline$h(\cdot)$ & A one-way hash function \\
\hline$\oplus$ & Exclusive OR operation \\
\hline$\|$ & Concatenation operation \\
\hline$A \rightarrow B: X$ & X is transmitted from A to B \\
\hline
\end{tabular}

\section{Registration Phase}

When a new user $U_{i}$ wants to register with $R C$, he/she performs the following steps:

Step R1. $U_{i} \rightarrow R C:\left\{I D_{i}, h\left(I D_{i} \| P W_{i}\right), h\left(b \oplus P W_{i}\right)\right\}$

$U_{i}$ selects identity $I D_{i}$ and password $P W_{i}$, and computes $h\left(I D_{i} \| P W_{i}\right)$ and $h\left(b \oplus P W_{i}\right)$, where $b$ is a random number generated by $U_{i}$. Then $U_{i}$ sends $I D_{i}$, $h\left(I D_{i} \| P W_{i}\right)$, and $h\left(b \oplus P W_{i}\right)$ to $R C$ for registration through a secure channel.

Step R2. $R C \rightarrow U_{i}:\left\{\operatorname{smart}\right.$ card $\left.\left[A_{i}, C_{i}, D_{i}, h(\cdot), h(y)\right]\right\}$

$R C$ computes the followings:

$A_{i}=h\left(h\left(I D_{i} \| P W_{i}\right) \| h\left(b \oplus P W_{i}\right)\right)$

$B_{i}=h\left(x \| I D_{i}\right)$

$D_{i}=h\left(I D_{i} \| P W_{i}\right) \oplus h\left(B_{i} \| h(x \| y)\right)$

$E_{i}=B_{i} \oplus h(x \| y)$.

Then, $R C$ issues a smart card containing $\left[A_{i}, D_{i}, E_{i}, h(\cdot), h(y)\right]$ and delivers it to $U_{i}$ through a secure channel.

Step R3. $U_{i}$ enters $b$ into his/her smart card and, and the smart card contains $\left[A_{i}, D_{i}, E_{i}, b, h(\cdot), h(y)\right]$.

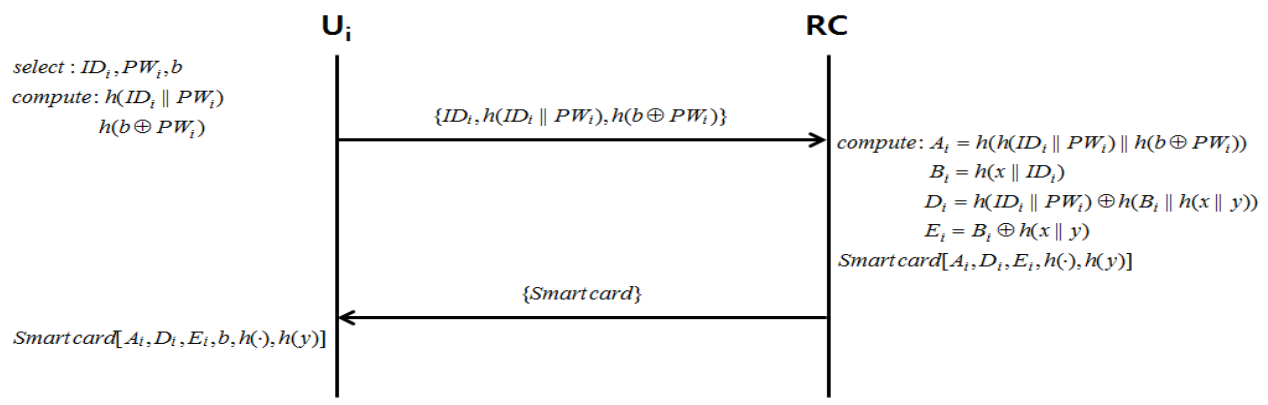

Figure 1. Registration Phase in our Scheme 


\section{Login Phase}

Step L1. $U_{i}$ inserts his/her smart card into the device and inputs identity $I D_{i}$ and password $P W_{i}$. The smart card computes $h\left(I D_{i} \| P W_{i}\right), h\left(b \oplus P W_{i}\right)$, and $A_{i}^{*}=$ $h\left(h\left(I D_{i} \| P W_{i}\right) \| h\left(b \oplus P W_{i}\right)\right)$ and checks whether $A_{i}^{*}$ is equal to $A_{i}$. If they are not equal, the procedure is terminated. Otherwise, $U_{i}$ proceeds the following steps.

Step L2. The smart card computes the followings:

$$
\begin{aligned}
& C I D_{i}=h\left(b \oplus P W_{i}\right) \oplus h\left(h\left(I D_{i} \| P W_{i}\right)\left\|S I D_{j}\right\| N_{i}\right) \\
& C_{1}=h\left(S I D_{j} \| h(y)\right) \oplus N_{i} \\
& C_{2}=h\left(h\left(S_{j} D_{j} \| h(y)\right) \| N_{i}\right) \oplus E_{i} \\
& C_{3}=h\left(E_{i}\left\|h\left(S I D_{j} \| h(y)\right)\right\| N_{i}\right) \oplus D_{i} \\
& C_{4}=h\left(h\left(b \oplus P W_{i}\right)\left\|C I D_{i}\right\| D_{i}\left\|E_{i}\right\| N_{i}\right),
\end{aligned}
$$

where $N_{i}$ is a random nonce generated by $U_{i}$.

Step L3. $U_{i} \rightarrow S_{j}:\left\{C I D_{i}, C_{1}, C_{2}, C_{3}, C_{4}\right\}$

$U_{i}$ sends $C I D_{i}, C_{1}, C_{2}, C_{3}$, and $C_{4}$ to $S_{j}$.

\section{Verification Phase}

Step V1. $S_{j}$ computes the followings:

$$
\begin{aligned}
& N_{i}=C_{1} \oplus h\left(S I D_{j} \| h(y)\right) \\
& E_{i}=C_{2} \oplus h\left(h\left(S I D_{j} \| h(y)\right) \| N_{i}\right) \\
& B_{i}=E_{i} \oplus h(x \| y) \\
& D_{i}=C_{3} \oplus h\left(E_{i}\left\|h\left(S I D_{j} \| h(y)\right)\right\| N_{i}\right) \\
& h\left(I D_{i} \| P W_{i}\right)=D_{i} \oplus h\left(B_{i} \| h(x \| y)\right) \\
& h\left(b \oplus P W_{i}\right)=C I D_{i} \oplus h\left(h\left(I D_{i} \| P W_{i}\right)\left\|S I D_{j}\right\| N_{i}\right) \\
& C_{4}^{\prime}=h\left(h\left(b \oplus P W_{i}\right)\left\|C I D_{i}\right\| D_{i}\left\|E_{i}\right\| N_{i}\right) .
\end{aligned}
$$

Step V2. $S_{j}$ checks whether $C_{4}^{\prime}$ is equal to $C_{4}$. If they are equal, $S_{j}$ generates random nonce $N_{j}$. Then, $S_{j}$ computes the followings:

$C_{5}=h\left(b \oplus P W_{i}\right) \oplus N_{i} \oplus N_{j}$

$C_{6}=h\left(h\left(b \oplus P W_{i}\right)\left\|h\left(I D_{i} \| P W_{i}\right)\right\| S I D_{j} \| N_{i}\right)$,

and sends $C_{5}$ and $C_{6}$ to $U_{i}$.

Step V3. $U_{i}$ computes the followings:

$N_{j}=C_{5} \oplus h\left(b \oplus P W_{i}\right) \oplus N_{i}$

$C_{6}^{\prime}=h\left(h\left(b \oplus P W_{i}\right)\left\|h\left(I D_{i} \| P W_{i}\right)\right\| S I D_{j} \| N_{i}\right)$,

and checks whether $C_{6}^{\prime}$ is equal to $C_{6}$. If they are equal, $U_{i}$ authenticates $S_{j}$. Then, $U_{i}$ computes $C_{7}=h\left(h\left(b \oplus P W_{i}\right)\left\|h\left(I D_{i} \| P W_{i}\right)\right\| S I D_{j} \| N_{j}\right)$ and sends $C_{7}$ to $S_{j}$.

Step V4. $S_{j} \quad$ computes $\quad C_{7}^{\prime}=h\left(h\left(b \oplus P W_{i}\right)\left\|h\left(I D_{i} \| P W_{i}\right)\right\| S I D_{j} \| N_{j}\right) \quad$ and checks whether $C_{7}^{\prime}$ is equal to $C_{7}$. If they are not equal, the procedure is terminated. Otherwise, $S_{j}$ authenticates $U_{i}$ and the mutual authentication is completed. 
After the mutual authentication, $U_{i}$ and $S_{j}$ computes $S K=h\left(h\left(b \oplus P W_{i}\right) \|\right.$ $\left.h\left(I D_{i} \| P W_{i}\right)\left\|S I D_{j}\right\| N_{i} \| N_{j}\right)$ for future secure communication.

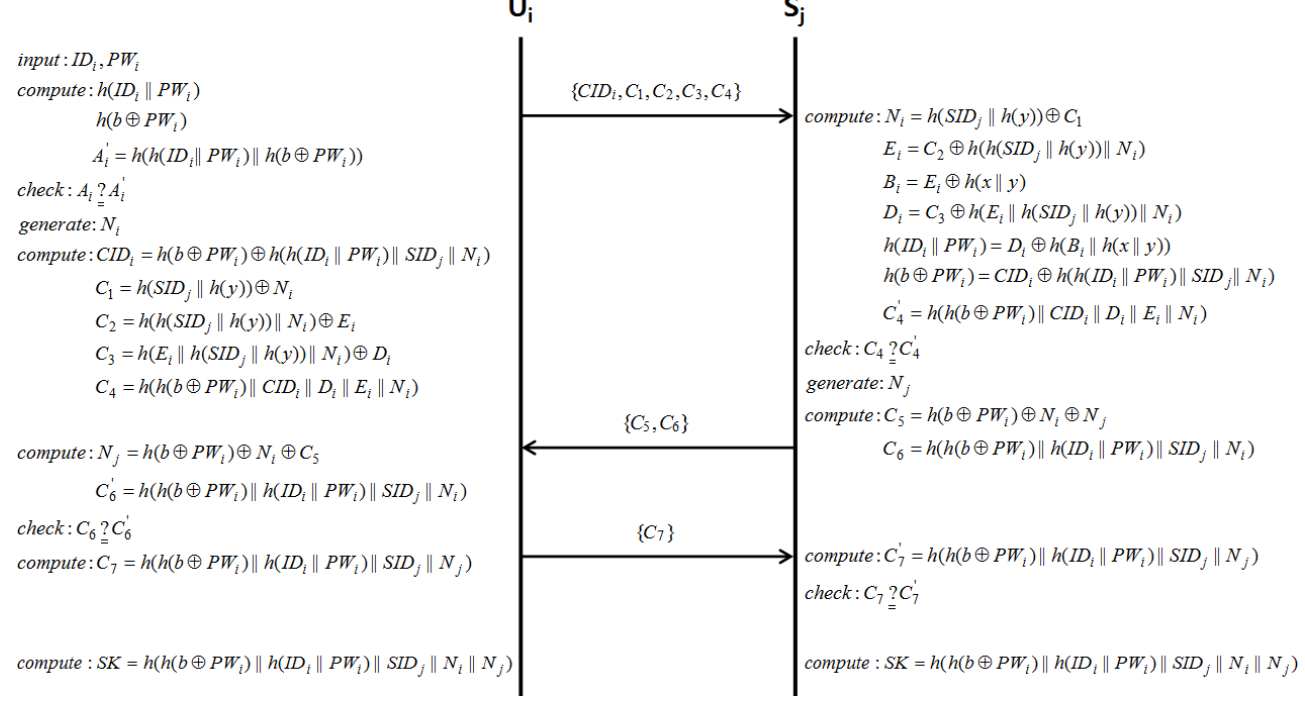

Figure 2. Login and Verification Phase in our Scheme

\section{Password Change Phase}

Step P1. $U_{i}$ inserts his/her smart card into the device and inputs identity $I D{ }_{i}$ and password $P W_{i}$.

Step P2. The smart card computes $h\left(I D_{i} \| P W_{i}\right), h\left(b \oplus P W_{i}\right)$, and $A_{i}^{*}=h\left(h\left(I D_{i} \| P W_{i}\right) \|\right.$ $\left.h\left(b \oplus P W_{i}\right)\right)$, and checks whether $A_{i}^{*}$ is equal to $A_{i}$. If they are not equal, the procedure is terminated. Otherwise, $U_{i}$ inputs a new password $P W_{i}{ }^{\text {new }}$ and a new random number $b^{\text {new }}$.

Step P3. The smart card computes $h\left(I D_{i} \| P W_{i}{ }^{\text {new }}\right), h\left(b^{\text {new }} \| P W_{i}{ }^{\text {new }}\right), A_{i}^{\text {new }}=h\left(h\left(I D_{i} \|\right.\right.$ $\left.\left.P W_{i}{ }^{\text {new }}\right) \| h\left(b^{\text {new }} \| P W_{i}^{\text {new }}\right)\right)$, and $D_{i}^{\text {new }}=D_{i} \oplus h\left(I D_{i} \| P W_{i}\right) \oplus h\left(I D_{i} \| P W_{i}{ }^{\text {new }}\right)$.

Step P4. Finally, the smart card replaces $A_{i}$ with $A_{i}^{\text {new }}$, and $D_{i}$ with $D_{i}^{\text {new }}$.

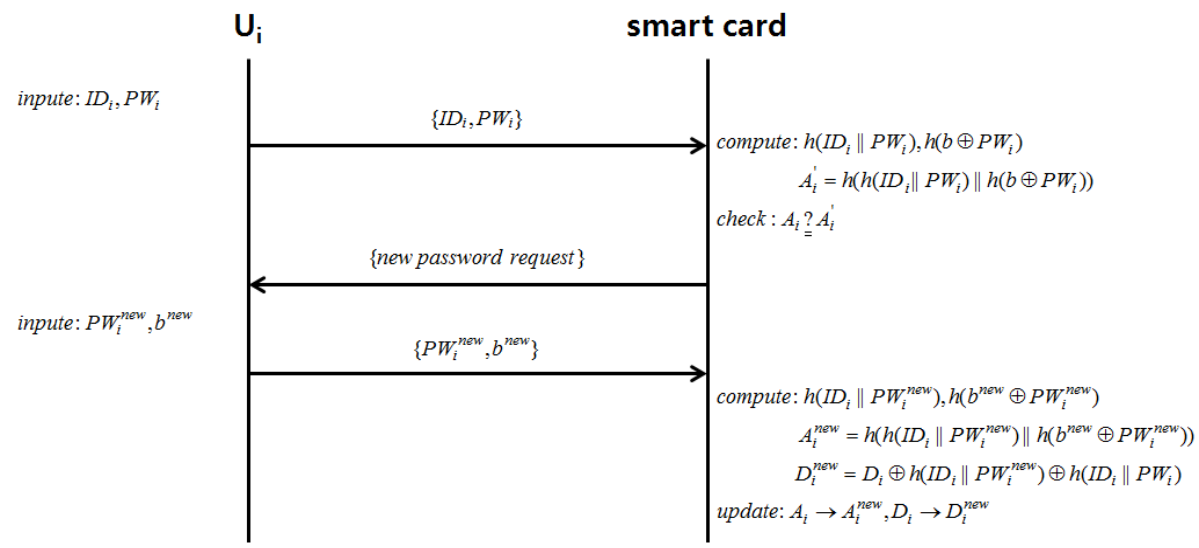

Figure 3. Password Change Phase in our Scheme 


\section{Analyses}

\section{Security Analysis}

Table 2 compares the security of existing schemes with our proposed scheme. Our scheme has the following security properties:

Known-key Secrecy: If an attacker obtains the session key $S K$ in the previous session, he/she cannot compute session key in later session. As the nature of a one-way hash function, an attacker cannot obtain $h\left(I D_{i} \| P W_{i}\right)$ and $h\left(b \oplus P W_{i}\right)$ in session key. Also if an attacker are stolen $U_{i}$ 's smart card or eavesdrops the previous message, he/she compute the session key from it.

Forward Secrecy: If an attacker obtains the master secret key $x$, he/she cannot compute the session key of the previous session. The reason cannot know $b, I D_{i}$, and $P W_{i}$. Although an attacker are stolen $U_{i}$ 's smart card or eavesdrops the previous message, he/she compute the previous session key without knowing $b, I D_{i}$, and $P W_{i}$.

Replay Attack: Assume that an attacker eavesdrops on the login request message $\left\{C I D_{i}, C_{1}, C_{2}, C_{3}, C_{4}, C_{5}, C_{6}, C_{7}\right\}$ for the previous session. An attacker is replaying the eavesdropping message to authenticate, but he/she cannot authenticate from the server.

Forgery Attack: Because an attacker cannot know $b, I D_{i}, P W_{i}, N_{i}, h(y), E_{i}$, and $D_{i}$, he/she cannot compute the login request message $\left\{C I D_{i}, C_{1}, C_{2}, C_{3}, C_{4}\right\}$. Although an attacker are a legitimate user of the system, he/she cannot compute the login request message because of without knowing $b, P W_{i}$, and $I D_{i}$. Even though an attacker also obtain user's smart card, he/she cannot compute the login request message because of without knowing $I D_{i}$ and $P W_{i}$.

Server Spoofing Attack and Registration Center Spoofing Attack: When an attacker masquerade as a legitimate server, he/she cannot obtain $h\left(I D_{i} \| P W_{i}\right)$ and $h\left(b \oplus P W_{i}\right)$ because of without knowing $h(x \| y)$. Also because a legitimate server cannot know $h(y)$, he/she cannot masquerade as the other server. An attacker cannot masquerade as a legitimate registration center because of without knowing $x$ and $y$.

Stolen Smart Card Attack: Assume that an attacker obtains $U_{i}$ 's smart card or extracts information of $U_{i}$ 's smart card. But an attacker cannot compute the login request message because of without knowing $I D_{i}$ and $P W_{i}$. Also an attacker cannot obtain $I D_{i}$ and $P W_{i}$ through the extracted information. The reason cannot obtain $I D_{i}$ and $P W_{i}$ from the extracted information by the nature of a one-way hash function.

Mutual Authentication: The user can authenticate the server by checking $C_{6}$ and the server can authenticate the user by checking $C_{7}$. 
Table 2. Security Analysis of the Compared Schemes

\begin{tabular}{|l|c|c|c|c|}
\hline & $\begin{array}{c}\text { Proposed } \\
\text { scheme }\end{array}$ & $\begin{array}{c}\text { Hsiang, } \text { et al. } \\
{[7]}\end{array}$ & $\begin{array}{c}\text { Lee, } \text { et al. } \\
{[8]}\end{array}$ & $\begin{array}{c}\text { Li, } \text { et al. } \\
{[9]}\end{array}$ \\
\hline Known-key secrecy & Yes & Yes & Yes & Yes \\
\hline Forward secrecy & Yes & Yes & Yes & Yes \\
\hline Replay attack & Yes & Yes & Yes & No \\
\hline Forgery attack & Yes & No & No & No \\
\hline $\begin{array}{l}\text { Server spoofing attack } \\
\text { and registration center } \\
\text { spoofing attack }\end{array}$ & Yes & No & No & Yes \\
\hline Stolen smart card attack & Yes & Yes & Yes & Yes \\
\hline Mutual authentication & Yes & No & No & Yes \\
\hline
\end{tabular}

\section{Performance Analysis}

Table 3 compares the performance of existing schemes with our proposed scheme. Figure 4 show the measured results for users performing the login and verification phase. As shown in Figure 4, our proposed scheme incurs less computational time than Hsiang, et al.'s scheme and incurs little more computational time as Lee, et al.'s scheme and Li, et $a l$. 's scheme. However our scheme is more secure against various attacks.

Table 3. Performance Analysis of the Compared Schemes

\begin{tabular}{|c|c|c|c|c|c|}
\hline \multicolumn{2}{|c|}{ Scheme } & $\begin{array}{c}\text { Proposed } \\
\text { scheme }\end{array}$ & $\begin{array}{c}\text { Hsiang, } \text { et al. } \\
{[7]}\end{array}$ & $\begin{array}{c}\text { Lee, } \text { et al. } \\
{[8]}\end{array}$ & $\begin{array}{c}\text { Li, } \text { et al. } \\
{[9]}\end{array}$ \\
\hline Registration & User & $2 \mathrm{~T}(\mathrm{~h})+1 \mathrm{~T}(\oplus)$ & $1 \mathrm{~T}(\mathrm{~h})+1 \mathrm{~T}(\oplus)$ & $1 \mathrm{~T}(\mathrm{~h})+1 \mathrm{~T}(\oplus)$ & $1 \mathrm{~T}(\mathrm{~h})+1 \mathrm{~T}(\oplus)$ \\
\cline { 2 - 6 } \begin{tabular}{c} 
Phase \\
\cline { 2 - 6 }
\end{tabular} & $\mathrm{RC}$ & $6 \mathrm{~T}(\mathrm{~T})+2 \mathrm{~T}(\oplus)$ & $5 \mathrm{~T}(\mathrm{~T})+4 \mathrm{~T}(\oplus)$ & $6 \mathrm{~T}(\mathrm{~T})+1 \mathrm{~T}(\oplus)$ & $6 \mathrm{~T}(\mathrm{~T})+1 \mathrm{~T}(\oplus)$ \\
\hline $\begin{array}{c}\text { Login \& } \\
\text { Verification } \\
\text { Phase }\end{array}$ & User & $11 \mathrm{~T}(\mathrm{~h})+7 \mathrm{~T}(\oplus)$ & $10 \mathrm{~T}(\mathrm{~h})+7 \mathrm{~T}(\oplus)$ & $10 \mathrm{~T}(\mathrm{~h})+4 \mathrm{~T}(\oplus)$ & $9 \mathrm{~T}(\mathrm{~h})+6 \mathrm{~T}(\oplus)$ \\
\cline { 2 - 6 } & Server & $8 \mathrm{~T}(\mathrm{~h})+8 \mathrm{~T}(\oplus)$ & $9 \mathrm{~T}(\mathrm{~h})+6 \mathrm{~T}(\oplus)$ & $8 \mathrm{~T}(\mathrm{~h})+2 \mathrm{~T}(\oplus)$ & $7 \mathrm{~T}(\mathrm{~h})+6 \mathrm{~T}(\oplus)$ \\
\hline \multicolumn{2}{|c|}{ Total (} & $\cdot$ & $3 \mathrm{~T}(\mathrm{~h})+6 \mathrm{~T}(\oplus)$ & $\cdot$ & $\cdot$ \\
\hline
\end{tabular}

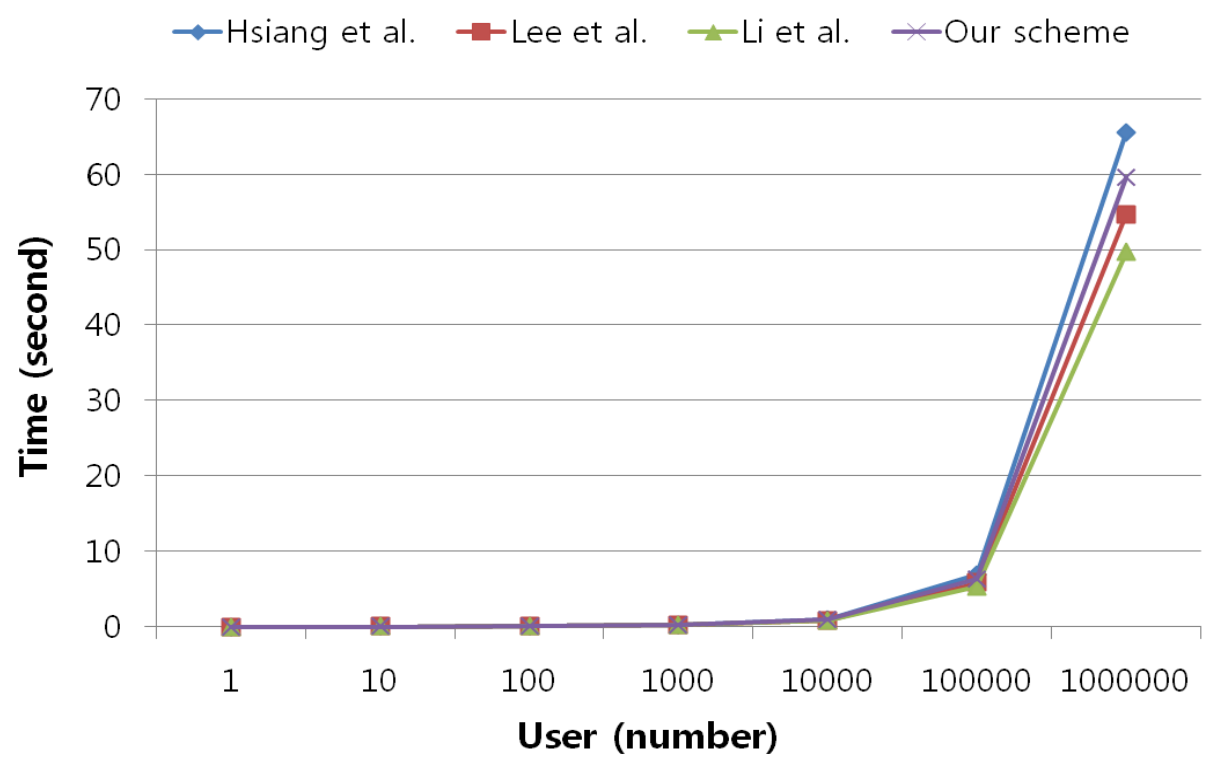

Figure 4. Analysis of Time According To Number of Users 


\section{Conclusions}

In this paper, we examined the security vulnerabilities of $\mathrm{Li}$, et al.'s scheme and proposed a security enhanced dynamic ID based remote user authentication scheme for multi-server environments. Our scheme provides mutual authentication and key establishment between user and server. In addition, our scheme has been proved that security to various attacks. Although our scheme incurs a little more computational costs than Lee, et al.'s scheme and Li, et al.'s scheme, it is more secure against various attacks than Lee, et al.'s scheme and Li, et al.'s scheme.

\section{Acknowledgments}

This work was supported by the National Research Foundation of Korea (NRF) grant funded by the Korea government (MSIP) (No. NRF-2014R1A2A1A11050818).

\section{References}

[1] W. Tsaur, "A Flexible User Authentication Scheme for Multi-server Internet Services”, Lecture Notes in Computer Science, vol. 2093, (2001), pp. 174-183.

[2] L. Li, I. Lin and M. Hwang, "A remote password authentication scheme for multiserver architecture using neural networks", IEEE Transactions on Neural Networks, vol. 12, no. 6, (2001), pp. 1498-1504.

[3] I. Lin, M. Hwang and L. Li, "A new remote user authentication scheme for multi-server architecture", Future Generation Computer Systems, vol. 19, no. 1, (2003), pp. 13-22.

[4] W. Juang, "Efficient multi-server password authenticated key agreement using smart cards", IEEE Transactions on Consumer Electronics, vol. 50, no. 1, (2004), pp. 251-255.

[5] C. Chang and J. Lee, "An efficient and secure multi-server password authentication scheme using smart cards", Proc. International Conference on Cyberworlds, (2004), pp. 417-422.

[6] Y. Liao and S. Wang, "A secure dynamic ID based remote user authentication scheme for multi-server environment", Computer Standards \& Interfaces, vol. 31, no. 1, (2009), pp. 24-29.

[7] H. Hsiang and W. Shih, "Improvement of the secure dynamic ID based remote user authentication scheme for multi-server environment", Computer Standards \& Interfaces, vol. 31, no. 6, (2009), pp. 1118-1123.

[8] C. Lee, T. Lin and R. Chang, "A secure dynamic ID based remote user authentication scheme for multiserver environment using smart cards", Expert Systems with Applications, vol. 38, no. 11, (2011), pp. 13863-13870.

[9] X. Li, J. Ma, W. Wang, Y. Xiong and J. Zhang, "A novel smart card and dynamic ID based remote user authentication scheme for multi-server environments", Mathematical and Computer Modelling, vol. 58, no. 1-2, (2013), pp. 85-95.

[10] C. Lee, Y. Lai and C. Li, "An Improved Secure Dynamic ID Based Remote User Authentication Scheme for Multi-Server Environment”, IJSIA, vol. 6, no. 2, (2012), pp. 203-209.

[11] C. Li, C. Weng and C. Fan, "Two-Factor User Authentication in Multi-Server Networks", IJSIA, vol. 6, no. 2, (2012), pp. 261-267.

[12] C. Li, C. Lee, H. Mei and C. Yang, "A Password and Smart Card Based User Authentication Mechanism for Multi-Server Environments”, IJFGCN, vol. 5, no. 4, (2012), pp. 153-163. 